osteological analysis of the type described above into the explanation of some of the major developments in New World prehistory.

All these studies show the importance of using the osteological evidence to make inferences about the human biological changes which have accompanied social and economic development, and which not only tell us more about those developments but may themselves have been responsible for them in certain circumstances, providing one of the 'kicks' which set positive feedback processes in motion.

\section{Sails set for the myosin active site}

\section{from Peter Chantler}

WHEREAS kinetic secrets are being prized almost daily from the myosin and actomyosin ATPase mechanisms knowledge of their structural aspects remains in the dark ages. The quest for structural information on the active site of myosin has been bedevilled by false leads. One example is that of the two thiol groups (SH-1 and SH-2) on each myosin head which were originally considered essential to the myosin ATPase mechanism, possibly being intimately involved with nucleotide at the active site. More recent studies however, have thrown doubt on the idea that these thiol groups are directly involved in the active site and a new twist to the story has recently emerged from Yount's laboratory at Washington State University. By an innovative technique in which the fate of radiolabelled nucleotide substrates was followed during chemical modification of myosin with cobalt, they have clarified some aspects of the relation of the thiol groups to the true active site and at last opened up a way for its unambiguous definition (Wells et al. Biochemistry 18, 4793; 4800; 1979; Wells \& Yount Proc. natn. Acad. Sci. U.S.A. 76, 4966; 1979).

Taking advantage of the exchange characteristics of $\mathrm{Co}$ (II) and $\mathrm{Co}$ (III) coordination complexes, myosin subfragment 1 (S-1) was exposed to a mixture of $\mathrm{CoCl}_{2}, 1,10$ phenanthroline and $\left[\mathrm{Co}^{\text {III }} \text { (phen) } \mathrm{CO}_{3}\right]^{+}$with a resultant inactivation of the ATPase activity (Wells et al. Biochemistry op. cit.) caused by tight chelation of one cobalt ion per mole S-1 which occurred in parallel with the loss of two sulphydryl groups. The mechanism proceeds by way of a S-1.Co ${ }^{\text {II }}$ (phen) intermediate ( $\mathrm{Co}^{\text {II }}$ freely exchangeable) which undergoes exchange with $\left[\mathrm{Co}^{\mathrm{III}}\right.$ (phen) $\left.{ }_{2} \mathrm{CO}_{3}\right]^{+}$to yield a S-1. CollI (phen) $x$ compound (CoII not exchangeable). Such in situ oxidation of the bound

Peter Chantler is at the Imperial Cancer Research Fund Laboratories, Lincoln's Inn, London.

\title{
Human teratocarcinoma workshop
}

Clinicians and mammalian embryologists are launching a concerted attack on human teratocarcinoma. The embryologists are moving on to this new challenge after some years spent manipulating mouse teratocarcinoma stem cell lines, obtained originally from tumours of the murine gonads. The value of such cell lines lies in the ease with which they can be induced to differentiate in a manner thought to be analogous to certain events of natural development. Although mouse development could hardly be said to be understood yet, insights into human development are now hoped for.

But embryologists are faced with the usual problems of working with human material. It is difficult to obtain, requiring the cooperation of surgeons who will ensure that a reasonable specimen is put aside before the whole tumour is claimed by a pathologist and doused in formalin. The problems of

cobalt is extremely mild; indeed, the S-1.Co ${ }^{I I I}$ (phen) $)_{x}$ can be reduced by short incubation with reagents such as Fe IIEDTA which fully restore enzymic activity. The simultaneous loss of $\mathrm{NH}_{4}{ }^{+}$-EDTA, $\mathrm{Ca}^{+}$and $\mathrm{Mg}^{+}-$ATPase activities on cobalt binding and the blocking of the binding by $p$-N,N'phenylenediamine (which crosslinks $\mathrm{SH}-1$ to SH-2) identified the two sulphydryls lost as SH-1 and SH-2 and showed that these two thiols could be cross-linked by the cobalt and therefore approach to within $3-5 \AA$ of each other (the distance depending on whether there was cis or trans chelation).

When the fate of ${ }^{14} \mathrm{C}$-labelled nucleotides was followed during the course of Co modification, inactivation of the ATPase amazingly paralleled incorporation of nucleotide so that complete inactivation occurred on simultaneous binding of one gram-atom of cobalt and one mole of nucleotide per mole S-1. This not only directly demonstrates the separate identities of the active site and the SH-1 and SH-2 thiols but also shows, consistent with other evidence, the interdependence of the thiol and active sites. When cobalt chelates SH-1 and SH-2, nucleotide at the active site is prevented from leaving; when nucleotide binds to the active site, cobalt chelates SH-1 and SH-2 much more easily. In addition, a new phrase enters the textbooks: active site trapping - a technique, in serendipitous cases, as here, of great potential power. When the experiment was performed in the presence of MgATP the trapped nucleotide always manifested itself as MgADP. Although the relationship between cobalt modification and nucleotide hydrolysis is unclear it is apparent from the relative rates of the two reactions that the trapped $\mathrm{ADP}$ did not originate from a rebinding of previously hydrolysed substrate. pathology and nomenclature are also formidable, and participants at a European workshop on human teratocarcinoma at Imperial Cancer Research Fund Laboratories (ICRF) in London on 28 January were not always sure what they were talking about. Nor were they all particularly confident of the difference between teratocarcinoma, seminoma and yolk sac tumour, although all three originate in the same anatomical region.

In future they will cooperate in obtaining cell lines and in characterising those already established. The workshop will gather again in the autum. Meanwhile information about the cell lines, their biochemical and immunological characteristics, is to be collected by Brigid Hogan of ICRF's Mill Hill laboratories for subsequent distribution. Later there may be a human teratocarcinoma newsletter.

M.L.

As active site trapping seems to be equally effective in the presence of adenosine $5^{\prime}-(\beta, \gamma$-imido)-triphosphate, a non-hydrolysable analogue of ATP, the technique may well offer some insight into mechanism. But its real future lies in conjunction with photoaffinity probes such as 8-azidoadenosine triphosphate which the authors have also trapped (Wells \& Yount op. cit.). The power of the photoaffinity probe, in more conventional mode, has been seen recently in the binding and incorporation of arylazido- $\beta$ -alanine-ATP into proteolytic subfragments of myosin followed by tryptic proteolysis (Szilagyi et al. Biochem. biophys. Res. Commun. 87, 936; 1979). The reagent labelled an N-terminal $25 \mathrm{~K}$ peptide of myosin; there was no incorporation into the fragment containing $\mathrm{SH}-1$ and SH-2. As this latter fragment is separated from the $25 \mathrm{~K}$ peptide by a $50 \mathrm{~K}$ peptide (Szilagyi et al. op. cit.) it is apparent that the sequentially remote 'essential' thiol region must be juxtaposed near to the active site in the tertiary structure of the enzyme.

Detailed speculations on which amino acids are involved at the active site of myosin are unwarranted but the most likely residue seems to be arginine (Mornet et al. Eur. J. Biochem. 100, 421; 1979), found at the heart of many nucleotide-binding enzymes (Riordan et al. Science 195, 884; 1977). Tryptophan, on the cards for many years becuase of the nucleotide-dependent tryptophan fluorescence enhancement of skeletal muscle myosin (Werber et al. Biochemistry, 11, 2872; 1972) seems less likely now in view of the absence of such effects in invertebrate myosins (Chantler \& Szent-Gyorgyi Biochemistry 17, $5440 ; 1978)$. Lysine, histidine and tyrosine have also been proposed. At last the opportunity to find out for certain is here. $\square$ 